\title{
TESTE DE LIXIVIAÇÃO DE POTÁSSIO PARA AVALIAÇÃO DO VIGOR DE SEMENTES DE AMENDOIM ${ }^{1}$
}

\author{
HAMILTON KIKUTI², PRISCILA FRATIN MEDINA ${ }^{3}$, ANA LÚCIA PEREIRA KIKUTI' ${ }^{4}$ NILZA PATRICIA RAMOS ${ }^{2}$
}

\begin{abstract}
RESUMO - O teste de lixiviação de potássio é baseado na integridade das membranas celulares das sementes, sendo considerado um procedimento rápido para a avaliação do vigor. O presente trabalho foi realizado no Instituto Agronômico (IAC) e na Escola Superior de Agricultura "Luiz de Queiroz"/Universidade de São Paulo (USP/ESALQ), com o objetivo de estabelecer procedimentos para o teste de lixiviação de potássio visando a avaliação do vigor de sementes de amendoim. Para a caracterização do potencial fisiológico dos lotes foram realizados os testes de germinação (velocidade, primeira contagem e total), de envelhecimento acelerado com uso de solução saturada de $\mathrm{NaCl}$, de emergência de plântulas em campo (velocidade e percentagem). Também foi determinado o grau de umidade das sementes. O teste de lixiviação de potássio foi realizado com amostras de 25 e 50 sementes, de 6 lotes do cultivar Runner IAC 886, colocadas em copos plásticos contendo 75, 100 e $150 \mathrm{~mL}$ de água destilada, à $25^{\circ} \mathrm{C}$. As leituras foram efetuadas em intervalos de 30 até 180 minutos Concluiu-se que o teste de lixiviação de potássio é eficiente em distinguir o vigor de lotes sementes de amendoim, a partir de 60 minutos de embebição. A combinação 25 sementes, 75 ou 100mL de água e 60 minutos de embebição foi considerada a melhor opção para a diferenciação do vigor, possibilitando maior agilidade na tomada de decisões durante programas de controle de qualidade conduzidos por empresas produtoras de sementes.
\end{abstract}

Termos para indexação: Arachis hypogaea, vigor, controle de qualidade, análise de sementes.

\section{POTASSIUM LEACHATE TEST TO ASSESS PEANUT SEED VIGOR}

\begin{abstract}
The potassium leachate test is based on the integrity of seed cellular membranes, and is considered a fast procedure to assess seed vigor. The present research was conducted at the Agronomic Institute - IAC and USP/ESALQ, Brazil, with the objective of establishing procedures for the potassium leachate test to assess peanut seed vigor. The physiological potential was characterized by germination (speed, first count and percentage), accelerated aging, field seedling emergence (speed and percentage). Seed moisture content was also determined. The potassium leachate test was carried out with samples of 25 and 50 seeds in six lots of the Runner IAC 886 cultivar, placed in plastic cups containing 75,100 and $150 \mathrm{~mL}$ of distilled water at $25^{\circ} \mathrm{C}$. The evaluations were performed at 30 minute intervals up to 180 minutes. It was concluded that the potassium leachate test was efficient to discriminate seed peanut lots of high and low vigor, after 60 minutes of imbibition. The imbibition of 25 -seed samples in 75 or $100 \mathrm{~mL}$ water for 60 minutes was considered the best alternative for discriminating the seed lot vigor in a short period of time.
\end{abstract}

Index terms: Arachis hypogaea, vigor, quality control, seeds analysis.

${ }^{1}$ Submetido em 11/01/2007. Aceito para publicação em 30/08/2007.

${ }^{2}$ Engenheiro Agrônomo, Dr., Pesquisador Científico, Instituto Agronômico,

IAC, Caixa Postal 28, 13020-902 Campinas (SP) e-mail: hkikuti@iac.sp.gov.br; pfmedina@iac.sp.gov.br; npramos@iac.sp.gov.br

${ }^{3}$ Engenheiro Agrônomo, Dra., Escola Superior de Agricultura "Luiz de Queiroz", ESALQ, Caixa Postal 9, 13418900 Piracicaba (SP) e-mail: alkikuti@esalq.usp.br 


\section{INTRODUÇÃO}

A cadeia produtiva do amendoim vem se modernizando e a idéia de racionalização dos fatores de produção, inclui os produtos químicos utilizados, conjuntamente com a rastreabilidade de atividades de manejo realizadas, além da consciência da necessidade de utilização de sementes certificadas por parte dos produtores que queiram continuar atuando nesta atividade, torna-se uma realidade.

A utilização de sementes certificadas é um dos itens exigidos pelo programa de produção integrada de amendoim (PIA); sistema de certificação de qualidade que está sendo proposto aos produtores de amendoim como diferencial de garantia de qualidade e ambientalmente correto. Neste contexto o aperfeiçoamento e o desenvolvimento de procedimentos rápidos e eficientes para a avaliação do potencial fisiológico das sementes de amendoim tornam-se importante.

O teste de germinação é fundamental para avaliação do potencial fisiológico das sementes, porém, este pode superestimar o desempenho em campo, por ser conduzido em condições ideais de temperatura e umidade.

Os testes de vigor têm sido usados em complementação às informações obtidas no teste de germinação e seus resultados se assemelham mais aos obtidos em campo que os do teste de germinação, principalmente quando as condições ambientais se desviam das mais favoráveis (Marcos Filho, 1999a).

O teste de lixiviação de potássio vem se destacando para avaliação do potencial fisiológico de sementes, produzindo resultados satisfatórios para várias espécies (Marcos Filho, 2005). Este teste tem princípio semelhante ao de condutividade elétrica, baseando-se na integridade das membranas celulares das sementes. A diferença entre eles é que, no de condutividade elétrica determina-se a quantidade total de íons liberados durante a embebição e, no de lixiviação de potássio, somente quantifica-se a quantidade de potássio lixiviado na solução, visto que este é o principal íon inorgânico lixiviado pelas sementes durante a embebição (Loomis \& Smith, 1980; Powell, 1986).

Não tem sido detectada a associação da quantidade de potássio lixiviado com os teores deste elemento nas sementes, confirmando que a liberação de potássio durante a embebição está diretamente associada à permeabilidade das membranas celulares (Custódio \& Marcos Filho, 1997).

Da mesma forma que ocorre no teste de condutividade elétrica, sementes menos vigorosas apresentam menor velocidade de estruturação das membranas quando embebidas em água, tendo como conseqüência maior liberação de exsudatos para o exterior da célula que aquelas mais vigorosas (Hampton \& TeKrony, 1995; Marcos Filho, 2005), conseqüentemente apresentando maior liberação de potássio.

Simon \& Raja-Harun (1972) afirmaram que os resultados obtidos no teste de lixiviação de potássio são comparáveis aos obtidos no teste de condutividade elétrica. No entanto, Woodstock et al. (1985) relataram que a liberação de potássio e cálcio proporcionou melhor indicação do potencial fisiológico de sementes de algodão quando comparado com a quantidade total de íons liberados pelas sementes, resultante da avaliação da condutividade elétrica. A redução no período de avaliação é outro ponto positivo observado no teste de lixiviação de potássio, sendo que após 30 minutos de embebição para sementes de feijão (Barros et al., 1999), milho (Miguel \& Marcos Filho, 2002) e soja (Custódio \& Marcos Filho, 1997), foi possível determinar diferenças entre lotes.

Quanto ao número de sementes, foram observados resultados promissores com o uso de 25, 50 e 100 sementes, dependendo da espécie (Vanzolini \& Nakagawa, 2003; Rodo \& Marcos Filho, 2001; Panobianco \& Marcos Filho, 2001). O volume de água também é variável, encontrando-se resultados consistentes com a utilização de $25 \mathrm{~mL}$ (Miranda et al., 2003; Panobianco \& Marcos Filho, 2001a) e 75mL (Vanzolini \& Nakagawa, 2003; Dias et al., 1997; Dias et al., 1998).

Resultados promissores com o uso do teste de lixiviação de potássio para identificar diferenças no potencial fisiológico de sementes de amendoim foram obtidos por Vanzolini \& Nakagawa (2003). Estes autores trabalharam com dois lotes do cultivar Tatu, utilizando quatro repetições de 25 sementes, embebidas em $75 \mathrm{~mL}$ de água destilada, a $20^{\circ} \mathrm{C}$, com avaliações realizadas a cada 3 horas, durante 30 horas de embebição e obtiveram resultados adequados já com a utilização do menor período de embebição.

No entanto, trabalhos de Rodo \& Marcos Filho (2001) com cebola, de Barros et al. (1999) com feijão, de Miguel \& Marcos Filho (2002) com milho, de Miranda et al. (2003) com pimentão e de Custódio \& Marcos Filho (1997) com soja, obtiveram resultados satisfatórios com a utilização de períodos de embebição de 30 a 60 minutos, destacando a necessidade de maiores estudos sobre este teste envolvendo a diminuição do tempo de embebição e variações nas combinações de número de sementes e volume de água de embebição, buscando-se a determinação dos procedimentos mais adequados para a sua condução.

Diante do exposto, o trabalho foi realizado com o objetivo de obter informações que permitam o aperfeiçoamento do teste de lixiviação de potássio para a avaliação do vigor de sementes de amendoim. 


\section{MATERIAL E MÉTODOS}

O trabalho foi realizado nos laboratórios de análise de sementes do Instituto Agronômico (IAC) e da Escola Superior de Agricultura "Luiz de Queiroz" - Universidade de São Paulo (USP/ESALQ). Foram utilizados seis lotes de sementes de amendoim, cultivar IAC Runner 886 , retidas na peneira 26 (crivos redondos), acondicionadas em sacos de papel e armazenadas em condições de ambiente controlado $\left(20^{\circ} \mathrm{C}\right.$ e $50 \%$ de umidade relativa do ar), durante todo o período experimental.

O teste de lixiviação de potássio foi realizado utilizandose diferentes combinações entre número de sementes e volumes de água $(25 / 75 \mathrm{~mL} ; 50 / 75 \mathrm{~mL} ; 25 / 100 \mathrm{~mL}, 50 / 100 \mathrm{~mL}$, $25 / 150 \mathrm{~mL}$ e $50 / 150 \mathrm{~mL}$ ) totalizando seis tratamentos repetidos quatro vezes. $\mathrm{O}$ teste foi repetido após 30 dias, para confirmação da adequação metodológica. Antes do início dos testes, as sementes foram pesadas $(0,0001 \mathrm{~g})$ e colocadas para embeber em copos plásticos (capacidade de 200 ou $300 \mathrm{~mL}$ ) contendo água destilada e mantidas em germinador, a $25^{\circ} \mathrm{C}$.

As leituras foram realizadas em fotômetro de chama DIGIMED NK-2004, a cada 30 minutos, até 180 minutos de embebição. O cálculo da lixiviação de potássio foi feito pela multiplicação da leitura obtida no fotômetro de chama (potássio/mL) pelo volume de água destilada $(\mathrm{mL})$ e dividido pela massa da amostra (g), sendo os resultados expressos em ppm de potássio/g de semente.

Para a caracterização do potencial fisiológico dos lotes foram utilizados os testes descritos a seguir.

A determinação do teor de água foi realizada, com duas subamostras de 25 sementes, para cada lote, pelo método da estufa, a $105^{\circ} \mathrm{C}$, durante 24 horas, de acordo com Brasil (1992). Esta determinação foi realizada no início das avaliações e após o envelhecimento acelerado com solução saturada de $\mathrm{NaCl}$.

O teste de germinação foi realizado com oito subamostras de 25 sementes para cada lote, tratadas com o fungicida Rhodiauram SC (3g de Thiram por $\mathrm{kg}$ de semente). Essas sementes foram distribuídas em rolos de papel toalha germitest, umedecidos com água destilada na proporção de 2,5 vezes a massa do substrato seco e mantidos a $25^{\circ} \mathrm{C}$. As avaliações foram realizadas no quinto e décimo dia após a instalação do teste, adotando-se os critérios estabelecidos em Brasil (1992).

A velocidade de germinação foi computada durante o teste de germinação, computando-se e retirando-se do substrato, diariamente, as plântulas normais a partir do início da germinação, até a sua estabilização. $O$ índice de velocidade de germinação foi calculado de acordo com a fórmula proposta por Maguire (1962). Registrou-se, também, a porcentagem de germinação no quarto dia, data estabelecida pelas R.A.S. (Brasil, 1992) para a primeira contagem desse teste.

O teste de emergência de plântulas foi realizado com quatro subamostras de 50 sementes para cada lote, distribuídas em bandejas de plástico, contendo terra + areia na proporção de $1: 1$, a $20^{\circ} \mathrm{C}$. A umidade inicial do substrato foi ajustada para $60 \%$ da capacidade de campo. A avaliação foi realizada aos dez dias após a semeadura. Nesse teste também foi avaliada a velocidade de emergência de plântulas, anotandose diariamente o número de plântulas que apresentavam as folhas embrionárias visíveis, até a estabilização. Ao final do teste, com os dados diários do número de plântulas normais, foi calculado o índice de velocidade de emergência, empregando-se a fórmula proposta por Maguire (1962).

$\mathrm{O}$ crescimento das plântulas foi realizado juntamente com o teste de emergência de plântulas. Aos 21 dias após a instalação do teste de emergência, as plântulas normais de cada repetição foram arrancadas, lavadas em água e avaliadas, com auxílio de uma régua, anotando-se o comprimento da parte aérea, do sistema radicular e o da plântula inteira. Os resultados foram expressos em mm plântula normal ${ }^{-1}$.

$\mathrm{O}$ teste de envelhecimento acelerado foi realizado com oito subamostras de 25 sementes, tratadas com Rhodiauram (Thiram), distribuídas numa camada única e uniforme, sobre tela de alumínio fixada em caixa de plástico (gerbox), contendo no fundo $40 \mathrm{~mL}$ de solução saturada de cloreto de sódio. As caixas plásticas foram mantidas a $42^{\circ} \mathrm{C}$, durante 72 horas (Marcos Filho, 1999b). Após esse período foi instalado o teste de germinação, de acordo com Brasil (1992). A avaliação foi realizada cinco dias após a instalação do teste de germinação.

Utilizou-se o delineamento inteiramente casualizado, com quatro ou oito repetições. As médias foram comparadas pelo teste de Tukey $(\mathrm{p} \leq 0,05)$.

\section{RESULTADOS E DISCUSSÃO}

As análises da variância dos dados obtidos nos testes de germinação, de emergência e de crescimento de plântulas de e de envelhecimento acelerado em solução saturada de cloreto de sódio, indicaram efeitos significativos de lotes.

De maneira geral, verificou-se que os lotes 3 e 5 destacaram-se dos demais, com maior nível de vigor (Tabela 1). No entanto, houve variação entre os testes, quanto à classificação dos lotes de menor vigor. 
TABELA 1. Resultados de germinação (G), primeira contagem da germinação (PCG), velocidade de germinação (VG), emergência de plântulas (EP), velocidade de emergência (VE), envelhecimento acelerado em solução saturada de $\mathrm{NaCl}$ (EASS), crescimento de plântulas (CP), teor de água inicial (TAI) e teor de água após o envelhecimento (TAE) em 6 lotes de sementes de amendoim da cultivar Runner IAC 886.

\begin{tabular}{clcccccccc}
\hline Lotes & G (\%) & PCG (\%) & VG & EP (\%) & VE & EASS $(\%)$ & CP $(\mathrm{cm})$ & TAI (\%) & TAE $(\%)$ \\
\hline 1 & $91 \mathrm{ab}$ & $87 \mathrm{ab}$ & $28,59 \mathrm{bc}$ & $73 \mathrm{abc}$ & $5,27 \mathrm{abc}$ & $79 \mathrm{~cd}$ & $61,5 \mathrm{bc}$ & 5,5 & 7,9 \\
2 & $90 \mathrm{ab}$ & $80 \mathrm{ab}$ & $27,61 \mathrm{c}$ & $62 \mathrm{c}$ & $4,27 \mathrm{c}$ & $84 \mathrm{bc}$ & $56,0 \mathrm{c}$ & 5,3 & 7,8 \\
3 & $98 \mathrm{a}$ & $95 \mathrm{a}$ & $31,99 \mathrm{a}$ & $85 \mathrm{a}$ & $6,09 \mathrm{a}$ & $95 \mathrm{a}$ & $82,0 \mathrm{ab}$ & 5,3 & 7,7 \\
4 & $82 \mathrm{~b}$ & $78 \mathrm{~b}$ & $30,84 \mathrm{ab}$ & $65 \mathrm{bc}$ & $4,80 \mathrm{bc}$ & $73 \mathrm{~d}$ & $58,5 \mathrm{c}$ & 5,3 & 7,7 \\
5 & $96 \mathrm{a}$ & $94 \mathrm{a}$ & $31,64 \mathrm{a}$ & $79 \mathrm{ab}$ & $5,60 \mathrm{ab}$ & $92 \mathrm{ab}$ & $86,0 \mathrm{a}$ & 5,4 & 7,8 \\
6 & $86 \mathrm{ab}$ & $76 \mathrm{~b}$ & $30,10 \mathrm{ab}$ & $62 \mathrm{c}$ & $4,52 \mathrm{c}$ & $84 \mathrm{bc}$ & $57,5 \mathrm{c}$ & 5,7 & 7,8 \\
\hline $\mathrm{CV}(\%)$ & 05,72 & 08,17 & 03,39 & 09,31 & 8,95 & 04,18 & 14,89 & & \\
\hline
\end{tabular}

Letras minúsculas: comparação dentro de cada coluna (Tukey, $\mathrm{p} \leq 0,05$ )

Pelo teste de germinação (primeira contagem e total) foi possível distinguir os lotes 3 e 5 como de maior vigor que o dos lotes 4 e 6 , na primeira contagem e, que o do lote 4 na porcentagem total de germinação.Todos os lotes apresentaram germinação superior a $80 \%$, valor mínimo estabelecido para a comercialização de sementes de amendoim. A utilização de lotes com elevado poder germinativo é recomendável para que sejam comparados os desempenhos de materiais compatíveis com as exigências estabelecidas para a comercialização (Powell, 1986)

Os lotes 2, 4 e 6 foram identificados como de menor nível de vigor que os lotes 3 e 5 pelo teste de crescimento de plântulas e o lote 3 pelos testes de emergência de plântulas (Tabela 1). Menor velocidade de germinação foi observada nas sementes dos lotes 1 e 2, em relação aos lotes 3 e 5; pelo teste de envelhecimento acelerado em solução saturada de cloreto de sódio, os lotes 1 e 4 foram menos vigorosos em relação aos lotes 3 e 5 e ainda, o lote 1 foi de vigor intermediário (Tabela 1).

O teor de água inicial das sementes variou de 5,3\% a 5,7\% (Tabela 1), sendo a diferença de apenas 0,4 pontos percentuais do lote de menor para o de maior teor de água. Também, após a incubação para o teste de envelhecimento acelerado, a variação entre o teor de água entre as amostras foi de apenas 0,2 pontos percentuais. Por esse resultado é possível afirmar que esse parâmetro não afetou os resultados dos testes de vigor, pois de acordo com Marcos Filho (1999b), diferenças inferiores a $2 \%$ não afetam os resultados dos testes de vigor.

No teste de lixiviação de potássio, observou-se efeito significativo de lotes em todas as combinações de número de sementes e de volume de água, em todos os períodos de embebição, nas duas épocas, com exceção da combinação 50 sementes e $75 \mathrm{~mL}$ de água, com avaliação aos 30 minutos de embebição, na primeira época.

$\mathrm{O}$ uso de 25 sementes proporcionou resultados similares aos obtidos com uso de 50 sementes, indicando que o uso de 25 sementes foi efetivo em classificar lotes de sementes de amendoim em diferentes níveis de vigor, pelo teste de lixiviação de potássio. Em outros trabalhos foram obtidos resultados satisfatórios com o uso de 25 sementes (Vanzolini \& Nakagawa, 2003; Dias et al., 1998; Miranda et al., 2003; Dias et al., 1997, Custódio \& Marcos Filho, 1997).

De maneira geral, verificou-se que a partir de 60 minutos de embebição, as combinações de número de sementes e de volume de água utilizadas, foram eficientes em destacar os lotes 3 e 5 como os de maior nível de vigor (Tabelas 2,3 e 4), de maneira semelhante ao que foi observado nos demais testes de vigor (Tabela 1). Esses resultados corroboram os obtidos por Dias et al (1997), nos quais os autores conseguiram classificar lotes de sementes de soja em diferentes níveis de vigor a partir de 60 minutos de embebição, a $30^{\circ} \mathrm{C}$. A rapidez na obtenção de resultados é muito importante em programas de controle de qualidade para agilizar as tomadas decisões.

No teste de lixiviação de potássio foi possível destacar, de maneira geral, o comportamento inferior dos lotes 1, 2, $4 \mathrm{e}$ 6 quando comparados aos lotes 3 e 5, principalmente quando considerados os menores volumes de água $(75$ e $100 \mathrm{~mL})$, sendo esses resultados semelhantes aos obtidos nos demais testes de vigor (Tabela 1). Esses resultados são coerentes, pois de acordo com Marcos Filho (2005), é esperado que os testes de vigor permitam distinguir com segurança os lotes de alto dos de baixo vigor e que as diferenças detectadas estejam relacionadas ao comportamento das sementes durante o armazenamento e após a semeadura. 
TABELA 2. Resultados de lixiviação de potássio (ppm $\mathrm{g}^{-1}$ ) em 6 lotes de sementes de amendoim da cultivar Runner IAC 886, avaliadas com 30, 60, 90, 120, 150 e 180 minutos de embebição em água, com 25 e 50 sementes e $75 \mathrm{~mL}$ de água, avaliadas em duas épocas de testes.

\begin{tabular}{|c|c|c|c|c|c|c|}
\hline \multirow{3}{*}{$\begin{array}{l}\text { Lotes } \\
25 \text { sem. }\end{array}$} & \multicolumn{6}{|c|}{ Tempos de avaliação (minutos) } \\
\hline & \multicolumn{2}{|c|}{30} & \multicolumn{2}{|c|}{60} & \multicolumn{2}{|c|}{90} \\
\hline & $1^{\mathrm{a}}$ aval. & $2^{\mathrm{a}}$ aval. & $1^{\mathrm{a}}$ aval. & $2^{\mathrm{a}}$ aval. & $1^{\mathrm{a}}$ aval. & $2^{\mathrm{a}}$ aval. \\
\hline 1 & $22,16 \mathrm{c}$ & $24,11 \mathrm{~b}$ & $29,85 \mathrm{~b}$ & $33,36 \mathrm{~b}$ & $38,25 \mathrm{~b}$ & $41,72 b$ \\
\hline 2 & $24,26 \mathrm{c}$ & $26,33 \mathrm{~b}$ & $35,22 \mathrm{~b}$ & $35,24 b$ & $44,08 \mathrm{~b}$ & $43,64 \mathrm{~b}$ \\
\hline 3 & $12,74 \mathrm{a}$ & $12,85 \mathrm{a}$ & $19,70 \mathrm{a}$ & $17,60 \mathrm{a}$ & $24,37 \mathrm{a}$ & $22,25 \mathrm{a}$ \\
\hline 4 & $23,81 \mathrm{c}$ & $23,86 \mathrm{~b}$ & $32,16 \mathrm{~b}$ & $31,67 \mathrm{~b}$ & $40,00 \mathrm{~b}$ & $40,62 \mathrm{~b}$ \\
\hline 5 & $13,08 \mathrm{ab}$ & $15,26 \mathrm{a}$ & $18,23 \mathrm{a}$ & $20,04 \mathrm{a}$ & $24,15 \mathrm{a}$ & $25,34 \mathrm{a}$ \\
\hline 6 & $20,64 \mathrm{bc}$ & $24,15 \mathrm{~b}$ & $30,90 \mathrm{~b}$ & $32,27 \mathrm{~b}$ & $39,43 \mathrm{~b}$ & $42,14 \mathrm{~b}$ \\
\hline $\mathrm{CV}(\%)$ & 17,67 & 10,88 & 10,73 & 08,71 & 09,48 & 28,41 \\
\hline 50 sem. & $1^{\mathrm{a}}$ aval. & $2^{\mathrm{a}}$ aval. & $1^{\mathrm{a}}$ aval. & $2^{\mathrm{a}}$ aval. & $1^{\mathrm{a}}$ aval. & $2^{\mathrm{a}}$ aval. \\
\hline 1 & $23,03 \mathrm{a}$ & $21,70 \mathrm{~b}$ & $29,75 \mathrm{~b}$ & $26,61 \mathrm{~b}$ & $34,48 \mathrm{~b}$ & $35,10 \mathrm{~b}$ \\
\hline 2 & $27,25 \mathrm{a}$ & $21,61 \mathrm{~b}$ & $26,91 \mathrm{~b}$ & $29,48 \mathrm{~b}$ & $37,50 \mathrm{~b}$ & $39,03 \mathrm{~b}$ \\
\hline 3 & $12,06 \mathrm{a}$ & $13,13 \mathrm{a}$ & $18,13 \mathrm{a}$ & $18,23 \mathrm{a}$ & $20,97 \mathrm{a}$ & $22,91 \mathrm{a}$ \\
\hline 4 & $23,75 \mathrm{a}$ & $20,91 \mathrm{~b}$ & $30,62 \mathrm{~b}$ & $28,84 \mathrm{~b}$ & $37,07 \mathrm{~b}$ & $38,92 \mathrm{~b}$ \\
\hline 5 & $12,93 \mathrm{a}$ & $12,97 \mathrm{a}$ & $18,08 \mathrm{a}$ & 18,65 a & $21,55 \mathrm{a}$ & $24,13 \mathrm{a}$ \\
\hline 6 & $27,85 \mathrm{a}$ & $18,92 \mathrm{~b}$ & $24,84 \mathrm{ab}$ & $26,84 \mathrm{~b}$ & $33,30 \mathrm{~b}$ & $35,50 \mathrm{~b}$ \\
\hline $\mathrm{CV}(\%)$ & 42,46 & 09,85 & 13,67 & 07,71 & 13,36 & 07,70 \\
\hline
\end{tabular}

\begin{tabular}{|c|c|c|c|c|c|c|}
\hline \multirow{3}{*}{$\begin{array}{l}\text { Lotes } \\
25 \text { sem. }\end{array}$} & \multicolumn{6}{|c|}{ Tempos de avaliação (minutos) } \\
\hline & \multicolumn{2}{|c|}{120} & \multicolumn{2}{|c|}{150} & \multicolumn{2}{|c|}{180} \\
\hline & $1^{\mathrm{a}}$ aval. & $2^{\mathrm{a}}$ aval. & $1^{\mathrm{a}}$ aval. & $2^{\mathrm{a}}$ aval. & $1^{\mathrm{a}}$ aval. & $2^{\mathrm{a}}$ aval. \\
\hline 1 & $45,94 \mathrm{~b}$ & $48,48 \mathrm{~b}$ & $52,68 \mathrm{~b}$ & $54,95 \mathrm{~b}$ & $59,46 \mathrm{bc}$ & $62,82 \mathrm{~b}$ \\
\hline 2 & $53,16 \mathrm{~b}$ & $51,40 \mathrm{~b}$ & $61,51 \mathrm{~b}$ & $57,81 \mathrm{~b}$ & $68,87 \mathrm{c}$ & $68,01 \mathrm{~b}$ \\
\hline 3 & $29,45 \mathrm{a}$ & $27,60 \mathrm{a}$ & $33,49 \mathrm{a}$ & $29,45 \mathrm{a}$ & $36,29 \mathrm{a}$ & $33,21 \mathrm{a}$ \\
\hline 4 & $49,83 \mathrm{~b}$ & $48,96 \mathrm{~b}$ & $57,75 \mathrm{~b}$ & $54,90 \mathrm{~b}$ & $63,39 \mathrm{bc}$ & $65,10 \mathrm{~b}$ \\
\hline 5 & $28,67 \mathrm{a}$ & $29,91 \mathrm{a}$ & $33,10 \mathrm{a}$ & $33,44 \mathrm{a}$ & $36,47 \mathrm{a}$ & $36,97 \mathrm{a}$ \\
\hline 6 & $47,73 \mathrm{~b}$ & $49,63 \mathrm{~b}$ & $59,20 \mathrm{~b}$ & $58,36 \mathrm{~b}$ & $49,61 \mathrm{ab}$ & $65,55 \mathrm{~b}$ \\
\hline $\mathrm{CV}(\%)$ & 09,61 & 07,52 & 09,47 & 07,63 & 13,56 & 08,15 \\
\hline 50 sem. & $1^{\mathrm{a}}$ aval. & $2^{\mathrm{a}}$ aval. & $1^{\mathrm{a}}$ aval. & $2^{\mathrm{a}}$ aval. & $1^{\mathrm{a}}$ aval. & $2^{\mathrm{a}}$ aval. \\
\hline 1 & $43,34 \mathrm{~b}$ & $42,51 \mathrm{~b}$ & $49,42 \mathrm{c}$ & $49,00 \mathrm{~b}$ & $60,06 \mathrm{~b}$ & $57,10 \mathrm{~b}$ \\
\hline 2 & $44,04 \mathrm{~b}$ & $45,76 \mathrm{~b}$ & $46,67 \mathrm{bc}$ & $52,61 \mathrm{~b}$ & $63,37 \mathrm{~b}$ & $61,65 \mathrm{~b}$ \\
\hline 3 & $25,76 \mathrm{a}$ & $27,72 \mathrm{a}$ & $29,40 \mathrm{a}$ & $30,82 \mathrm{a}$ & $31,87 \mathrm{a}$ & $35,28 \mathrm{a}$ \\
\hline 4 & $45,30 \mathrm{~b}$ & $47,72 \mathrm{~b}$ & $57,17 \mathrm{c}$ & $55,29 \mathrm{~b}$ & $63,58 \mathrm{~b}$ & $67,05 \mathrm{~b}$ \\
\hline 5 & $26,43 \mathrm{a}$ & $28,21 \mathrm{a}$ & $33,24 \mathrm{ab}$ & $31,62 \mathrm{a}$ & $38,45 \mathrm{a}$ & $34,83 \mathrm{a}$ \\
\hline 6 & $44,38 \mathrm{~b}$ & $43,52 \mathrm{~b}$ & $51,70 \mathrm{c}$ & $51,75 \mathrm{~b}$ & $60,64 \mathrm{~b}$ & $59,41 \mathrm{~b}$ \\
\hline $\mathrm{CV}(\%)$ & 11,18 & 07,30 & 15,94 & 09,11 & 14,48 & 09,40 \\
\hline
\end{tabular}

Letras minúsculas: comparação dentro de cada coluna (Tukey, $\mathrm{p} \leq 0,05$ ) 
TABELA 3. Resultados de lixiviação de potássio (ppm $\mathrm{g}^{-1}$ ) em 6 lotes de sementes de amendoim da cultivar Runner IAC 886, avaliadas com 30, 60, 90, 120, 150 e 180 minutos de embebição em água, com 25 e 50 sementes e $100 \mathrm{~mL}$ de água, avaliadas em duas épocas de testes.

\begin{tabular}{|c|c|c|c|c|c|c|}
\hline \multirow{3}{*}{$\begin{array}{l}\text { Lotes } \\
25 \text { sem. }\end{array}$} & \multicolumn{6}{|c|}{ Tempos de avaliação (minutos) } \\
\hline & \multicolumn{2}{|c|}{30} & \multicolumn{2}{|c|}{60} & \multicolumn{2}{|c|}{90} \\
\hline & $1^{\mathrm{a}}$ aval. & $2^{\mathrm{a}}$ aval. & $1^{\mathrm{a}}$ aval. & $2^{\mathrm{a}}$ aval. & $1^{\mathrm{a}}$ aval. & $2^{\mathrm{a}}$ aval. \\
\hline 1 & $22,42 \mathrm{c}$ & $14,46 \mathrm{ab}$ & $29,54 \mathrm{bc}$ & $27,48 \mathrm{~cd}$ & $42,04 \mathrm{bc}$ & $39,05 \mathrm{~b}$ \\
\hline 2 & $22,69 \mathrm{c}$ & $19,98 \mathrm{~b}$ & $33,18 \mathrm{c}$ & $32,16 \mathrm{~d}$ & $45,34 \mathrm{c}$ & $45,26 \mathrm{~b}$ \\
\hline 3 & $12,84 \mathrm{a}$ & $09,61 \mathrm{a}$ & $17,91 \mathrm{a}$ & $14,82 \mathrm{a}$ & $24,34 \mathrm{a}$ & $25,43 \mathrm{a}$ \\
\hline 4 & $18,48 \mathrm{bc}$ & $12,89 \mathrm{a}$ & $25,61 \mathrm{~b}$ & $25,86 \mathrm{bc}$ & $36,83 \mathrm{bc}$ & $40,88 \mathrm{~b}$ \\
\hline 5 & $12,04 \mathrm{a}$ & $14,12 \mathrm{ab}$ & $17,62 \mathrm{a}$ & $20,68 a b$ & $24,32 \mathrm{a}$ & $28,36 \mathrm{a}$ \\
\hline 6 & $16,24 \mathrm{ab}$ & $20,14 b$ & $24,95 \mathrm{ab}$ & $29,91 \mathrm{~cd}$ & $34,31 \mathrm{~b}$ & $41,49 \mathrm{~b}$ \\
\hline $\mathrm{CV}(\%)$ & 13,48 & 18,03 & 13,27 & 09,42 & 11,98 & 09,79 \\
\hline 50 sem. & $1^{\mathrm{a}}$ aval. & $2^{\mathrm{a}}$ aval. & $1^{\mathrm{a}}$ aval. & $2^{\mathrm{a}}$ aval. & $1^{\mathrm{a}}$ aval. & $2^{\mathrm{a}}$ aval. \\
\hline 1 & $18,88 \mathrm{c}$ & $15,72 \mathrm{abc}$ & $25,39 \mathrm{~b}$ & $28,46 \mathrm{~b}$ & $38,58 \mathrm{~b}$ & $47,19 \mathrm{~b}$ \\
\hline 2 & $20,00 \mathrm{c}$ & $18,72 \mathrm{bc}$ & $27,10 \mathrm{~b}$ & $30,70 \mathrm{~b}$ & $37,88 \mathrm{~b}$ & $40,01 \mathrm{~b}$ \\
\hline 3 & $10,16 \mathrm{a}$ & $10,88 \mathrm{a}$ & $14,48 \mathrm{a}$ & $16,30 \mathrm{a}$ & $21,21 \mathrm{a}$ & $23,15 \mathrm{a}$ \\
\hline 4 & $17,12 \mathrm{bc}$ & $19,18 \mathrm{c}$ & $22,17 b$ & $24,78 \mathrm{ab}$ & $31,25 \mathrm{~b}$ & $35,20 \mathrm{ab}$ \\
\hline 5 & $11,12 \mathrm{a}$ & $12,00 \mathrm{ab}$ & $15,20 \mathrm{a}$ & $16,65 \mathrm{a}$ & $21,01 \mathrm{a}$ & 22,99 a \\
\hline 6 & $13,56 \mathrm{ab}$ & $17,47 \mathrm{abc}$ & $22,49 \mathrm{~b}$ & $24,63 \mathrm{ab}$ & $31,12 \mathrm{~b}$ & $34,46 \mathrm{ab}$ \\
\hline $\mathrm{CV}(\%)$ & 12,65 & 20,34 & 11,25 & 02 & 12,29 & \\
\hline \multirow{2}{*}{ Lotes } & \multicolumn{6}{|c|}{ Tempos de avaliação (minutos) } \\
\hline & \multicolumn{2}{|c|}{120} & \multicolumn{2}{|c|}{150} & \multicolumn{2}{|c|}{180} \\
\hline $25 \mathrm{sem}$. & $1^{\mathrm{a}}$ aval. & $2^{\mathrm{a}}$ aval. & $1^{\mathrm{a}}$ aval. & $2^{\mathrm{a}}$ aval. & $1^{\mathrm{a}}$ aval. & $2^{\mathrm{a}}$ aval. \\
\hline 1 & $50,36 \mathrm{bc}$ & $49,23 \mathrm{~b}$ & $58,28 \mathrm{~b}$ & $57,48 \mathrm{~b}$ & $65,13 \mathrm{~b}$ & $64,12 \mathrm{~b}$ \\
\hline 2 & $53,62 \mathrm{c}$ & $54,52 \mathrm{~b}$ & $60,37 \mathrm{~b}$ & $64,33 \mathrm{~b}$ & $66,74 \mathrm{~b}$ & $72,04 \mathrm{~b}$ \\
\hline 3 & 29,39 a & $30,11 \mathrm{a}$ & $32,54 \mathrm{a}$ & $34,51 \mathrm{a}$ & $36,09 \mathrm{a}$ & $38,23 \mathrm{a}$ \\
\hline 4 & $45,93 \mathrm{bc}$ & $49,02 \mathrm{~b}$ & $53,19 b$ & $57,72 \mathrm{~b}$ & $57,31 \mathrm{~b}$ & $66,18 \mathrm{~b}$ \\
\hline 5 & $28,81 \mathrm{a}$ & $33,68 \mathrm{a}$ & $35,53 \mathrm{a}$ & $38,16 \mathrm{a}$ & $38,04 \mathrm{a}$ & $41,51 \mathrm{a}$ \\
\hline 6 & $42,15 \mathrm{~b}$ & $50,59 \mathrm{~b}$ & $52,23 \mathrm{~b}$ & $59,83 \mathrm{~b}$ & $56,85 \mathrm{~b}$ & $67,42 \mathrm{~b}$ \\
\hline $\mathrm{CV}(\%)$ & 11,85 & 07,25 & 11,55 & 07,21 & 14,17 & 07,72 \\
\hline 50sem. & $1^{\mathrm{a}}$ aval. & $2^{\mathrm{a}}$ aval. & $1^{\mathrm{a}}$ aval. & $2^{\mathrm{a}}$ aval. & $1^{\mathrm{a}}$ aval. & $2^{\mathrm{a}}$ aval. \\
\hline 1 & $45,58 \mathrm{~b}$ & $45,66 \mathrm{~b}$ & $58,32 \mathrm{~b}$ & $53,37 \mathrm{c}$ & $68,03 \mathrm{c}$ & $64,38 \mathrm{~b}$ \\
\hline 2 & $44,85 \mathrm{~b}$ & $47,54 \mathrm{~b}$ & $58,00 \mathrm{~b}$ & $56,86 \mathrm{c}$ & $62,23 \mathrm{bc}$ & $65,20 \mathrm{~b}$ \\
\hline 3 & $24,75 \mathrm{a}$ & $27,76 \mathrm{a}$ & $30,35 \mathrm{a}$ & $32,22 \mathrm{ab}$ & $31,46 \mathrm{a}$ & $35,78 \mathrm{a}$ \\
\hline 4 & $38,94 \mathrm{~b}$ & $41,36 \mathrm{ab}$ & $49,15 \mathrm{~b}$ & $48,73 \mathrm{bc}$ & $55,08 \mathrm{bc}$ & $57,38 \mathrm{ab}$ \\
\hline 5 & $25,83 \mathrm{a}$ & $27,02 \mathrm{a}$ & $31,57 \mathrm{a}$ & $31,25 \mathrm{a}$ & $32,95 \mathrm{a}$ & $35,14 \mathrm{a}$ \\
\hline 6 & $40,83 \mathrm{~b}$ & $40,93 \mathrm{ab}$ & $49,40 \mathrm{~b}$ & $48,44 \mathrm{bc}$ & $54,67 \mathrm{~b}$ & $58,97 \mathrm{~b}$ \\
\hline $\mathrm{CV}(\%)$ & 10,42 & 17,62 & 12,22 & 16,91 & 11,53 & 18,78 \\
\hline
\end{tabular}

Letras minúsculas: comparação dentro de cada coluna (Tukey, $\mathrm{p} \leq 0,05$ ) 


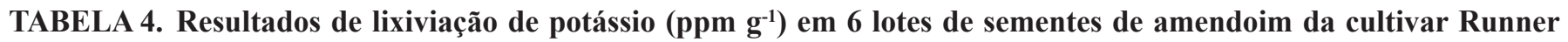
IAC 886, avaliadas com 30, 60, 90, 120, 150 e 180 minutos de embebição em água, com 25 e 50 sementes e $150 \mathrm{~mL}$ de água, avaliadas em épocas de testes.

\begin{tabular}{|c|c|c|c|c|c|c|}
\hline \multirow{3}{*}{$\begin{array}{l}\text { Lotes } \\
25 \mathrm{sem} .\end{array}$} & \multicolumn{6}{|c|}{ Tempos de avaliação (minutos) } \\
\hline & \multicolumn{2}{|c|}{30} & \multicolumn{2}{|c|}{60} & \multicolumn{2}{|c|}{90} \\
\hline & $1^{\mathrm{a}}$ aval. & $2^{\mathrm{a}}$ aval. & $1^{\mathrm{a}}$ aval. & $2^{\mathrm{a}}$ aval. & $1^{\mathrm{a}}$ aval. & $2^{\mathrm{a}}$ aval. \\
\hline 1 & $14,09 \mathrm{~b}$ & $20,88 \mathrm{c}$ & $21,79 \mathrm{ab}$ & $31,85 \mathrm{bc}$ & $35,31 \mathrm{a}$ & $47,44 \mathrm{~b}$ \\
\hline 2 & $20,62 \mathrm{c}$ & $24,57 \mathrm{c}$ & $29,46 \mathrm{~b}$ & $40,39 \mathrm{c}$ & $48,83 \mathrm{~b}$ & $49,46 \mathrm{~b}$ \\
\hline 3 & $11,48 \mathrm{ab}$ & $11,48 \mathrm{ab}$ & $17,37 \mathrm{a}$ & $20,28 \mathrm{a}$ & $24,69 \mathrm{a}$ & $26,15 \mathrm{a}$ \\
\hline 4 & $12,27 \mathrm{ab}$ & $20,85 \mathrm{c}$ & $23,04 \mathrm{ab}$ & $31,63 \mathrm{bc}$ & $32,76 \mathrm{a}$ & $44,21 \mathrm{~b}$ \\
\hline 5 & $08,77 \mathrm{a}$ & $09,02 \mathrm{a}$ & $17,31 \mathrm{a}$ & $14,77 \mathrm{a}$ & $24,73 \mathrm{a}$ & $26,43 \mathrm{a}$ \\
\hline 6 & $14,29 \mathrm{~b}$ & $18,48 \mathrm{bc}$ & $26,46 \mathrm{~b}$ & $26,07 \mathrm{ab}$ & $33,78 \mathrm{a}$ & $39,81 \mathrm{~b}$ \\
\hline $\mathrm{CV}(\%)$ & 17,28 & 20,26 & 17,54 & 15,58 & 17,92 & 11,46 \\
\hline 50sem. & $1^{\mathrm{a}}$ aval. & $2^{\mathrm{a}}$ aval. & $1^{\mathrm{a}}$ aval. & $2^{\mathrm{a}}$ aval. & $1^{\mathrm{a}}$ aval. & $2^{\mathrm{a}}$ aval. \\
\hline 1 & $16,62 \mathrm{~b}$ & $21,90 \mathrm{c}$ & $25,23 \mathrm{~b}$ & $29,82 \mathrm{~b}$ & $35,03 \mathrm{~b}$ & $40,41 \mathrm{bc}$ \\
\hline 2 & $15,70 \mathrm{~b}$ & $22,15 \mathrm{c}$ & $27,88 \mathrm{~b}$ & $33,53 \mathrm{~b}$ & $35,98 \mathrm{~b}$ & $43,81 \mathrm{c}$ \\
\hline 3 & $08,64 \mathrm{a}$ & $11,94 \mathrm{a}$ & $14,07 \mathrm{a}$ & $17,69 \mathrm{a}$ & $21,85 \mathrm{a}$ & $26,00 \mathrm{a}$ \\
\hline 4 & $11,61 \mathrm{ab}$ & $16,59 \mathrm{abc}$ & $20,94 \mathrm{ab}$ & $28,47 \mathrm{~b}$ & $34,24 \mathrm{~b}$ & $36,91 \mathrm{ab}$ \\
\hline 5 & $09,35 \mathrm{a}$ & $12,34 \mathrm{ab}$ & $16,80 \mathrm{a}$ & $18,83 \mathrm{a}$ & $22,95 \mathrm{a}$ & $26,77 \mathrm{a}$ \\
\hline 6 & $12,60 \mathrm{ab}$ & $20,01 \mathrm{bc}$ & $22,63 \mathrm{ab}$ & $28,77 \mathrm{~b}$ & $29,27 \mathrm{ab}$ & $35,02 \mathrm{~b}$ \\
\hline $\mathrm{CV}(\%)$ & 21,69 & 20,05 & 16,76 & 10,64 & 13,61 & 08,81 \\
\hline
\end{tabular}

\begin{tabular}{|c|c|c|c|c|c|c|}
\hline \multirow{3}{*}{$\begin{array}{l}\text { Lotes } \\
25 \text { sem. }\end{array}$} & \multicolumn{6}{|c|}{ Tempos de avaliação (minutos) } \\
\hline & \multicolumn{2}{|c|}{120} & \multicolumn{2}{|c|}{150} & \multicolumn{2}{|c|}{180} \\
\hline & $1^{\mathrm{a}}$ aval. & $2^{\mathrm{a}}$ aval. & $1^{\mathrm{a}}$ aval. & $2^{\mathrm{a}}$ aval. & $1^{\mathrm{a}}$ aval. & $2^{\mathrm{a}}$ aval. \\
\hline 1 & $44,02 \mathrm{bc}$ & $59,03 \mathrm{~b}$ & $51,77 \mathrm{ab}$ & $68,52 \mathrm{~b}$ & $60,37 \mathrm{~b}$ & $77,65 \mathrm{~b}$ \\
\hline 2 & $52,30 \mathrm{c}$ & $59,04 \mathrm{~b}$ & $61,91 \mathrm{~b}$ & $68,55 \mathrm{~b}$ & $70,92 \mathrm{~b}$ & $76,93 \mathrm{~b}$ \\
\hline 3 & $31,61 \mathrm{ab}$ & $31,81 \mathrm{a}$ & $37,05 \mathrm{a}$ & $36,84 \mathrm{a}$ & $41,46 \mathrm{a}$ & 39,36 a \\
\hline 4 & $43,36 \mathrm{abc}$ & $56,13 \mathrm{~b}$ & $51,38 \mathrm{ab}$ & $64,00 \mathrm{~b}$ & $60,06 \mathrm{~b}$ & $71,41 \mathrm{~b}$ \\
\hline 5 & $30,75 \mathrm{a}$ & $32,62 \mathrm{a}$ & $36,38 \mathrm{a}$ & $37,21 \mathrm{a}$ & $40,52 \mathrm{a}$ & $41,41 \mathrm{a}$ \\
\hline 6 & $47,30 \mathrm{c}$ & $50,08 \mathrm{~b}$ & $56,61 \mathrm{~b}$ & $61,84 \mathrm{~b}$ & $64,26 \mathrm{~b}$ & $71,20 \mathrm{~b}$ \\
\hline $\mathrm{CV}(\%)$ & 14,11 & 10,02 & 14,40 & 08,57 & 12,94 & 09,39 \\
\hline 50 sem. & $1^{\mathrm{a}}$ aval. & $2^{\mathrm{a}}$ aval. & $1^{\mathrm{a}}$ aval. & $2^{a}$ aval. & $1^{\mathrm{a}}$ aval. & $2^{\mathrm{a}}$ aval. \\
\hline 1 & $46,08 \mathrm{~b}$ & $49,36 \mathrm{bc}$ & $54,68 \mathrm{~b}$ & $58,69 \mathrm{~b}$ & $66,64 \mathrm{~b}$ & $69,37 \mathrm{~b}$ \\
\hline 2 & $45,38 \mathrm{~b}$ & $54,59 \mathrm{c}$ & $53,82 \mathrm{~b}$ & $63,62 \mathrm{~b}$ & $63,32 \mathrm{~b}$ & $70,21 \mathrm{~b}$ \\
\hline 3 & $28,04 \mathrm{a}$ & $30,52 \mathrm{a}$ & $33,27 \mathrm{a}$ & $33,70 \mathrm{a}$ & $37,43 \mathrm{a}$ & 36,99 a \\
\hline 4 & $42,96 \mathrm{ab}$ & $48,02 \mathrm{bc}$ & $49,83 \mathrm{ab}$ & $54,91 \mathrm{~b}$ & $58,42 \mathrm{ab}$ & $65,29 \mathrm{~b}$ \\
\hline 5 & 29,33 a & $31,16 \mathrm{a}$ & $33,89 \mathrm{a}$ & $35,76 \mathrm{a}$ & $37,60 \mathrm{a}$ & $39,21 \mathrm{a}$ \\
\hline 6 & $39,69 \mathrm{ab}$ & $44,34 \mathrm{~b}$ & $46,69 \mathrm{~b}$ & $53,99 \mathrm{~b}$ & $54,33 \mathrm{ab}$ & $60,41 \mathrm{~b}$ \\
\hline $\mathrm{CV}(\%)$ & 18,40 & 09,90 & 17,26 & 10,60 & 18,33 & 09,90 \\
\hline
\end{tabular}

Letras minúsculas: comparação dentro de cada coluna (Tukey, $\mathrm{p} \leq 0,05$ ) 
No teste de lixiviação de potássio, é possível separar lotes de sementes de amendoim em diferentes níveis de vigor, com a utilização de quaisquer combinações entre número de sementes e volumes de água estudados, a partir de 60 minutos de embebição. No entanto, a utilização de 25 sementes embebidas em 75 ou $100 \mathrm{~mL}$ de água durante 60 minutos, foram as melhores combinações por utilizar o menor número de sementes e permitir a separação de lotes de sementes de amendoim em níveis de vigor em curto período de tempo, possibilitando maior agilidade na tomada de decisão sobre o destino dos lotes, pelas empresas de sementes.

\section{CONCLUSÕES}

O teste de lixiviação de potássio realizado com a combinação de 25 sementes embebidas em 75 ou $100 \mathrm{~mL}$ de água por 60 minutos, constitui-se em opção eficiente para a diferenciação de lotes de sementes de amendoim quanto ao vigor.

\section{AGRADECIMENTOS}

Os autores agradecem aos Pesquisadores Científicos Roberto Tetsuo Tanaka e Ignácio José de Godoy e aos técnicos de apoio à pesquisa Denise Sayuri Ysa e Ivonete Alves dos Santos pelo apoio técnico e contribuições.

\section{REFERÊNCIAS}

BARROS, M.A.; OHSE, S.; MARCOS FILHO, J. Íon leakage as indicator of vigor in field bean seeds. Seed Technology, Lansing, v.21, n.1, p.44-48, 1999.

BRASIL. Ministério da Agricultura e Reforma Agrária. Regras para análise de sementes. Brasília: SNDA/DNDV/ CLAV, 1992. 365p.

CUSTÓDIO, C.C.; MARCOS FILHO, J. Potassium leachate test for the evaluation of soybean seed physiological quality. Seed Science and Technology, Zürich, v.25, n.3, p.549564, 1997.

DELOUCHE, J.C.; BASKIN, C.C. Acelerated aging techniques for predicting the relative storability of seed lots. Seed Science and Technology, Zürich, v.1, n.2, p.427-452, 1973.

DIAS, D.C.F.S.; MARCOS FILHO, J.; CARMELLO, Q.A.C. Potassium leakage test for the evaluation of vigour in soybean seeds. Seed Science and Technology, v.25, n.1, p.7-18, 1997.
DIAS, D.C.F.S.; VIEIRA, A.N.; BHÉRING, M.C. Condutividade elétrica e lixiviação de potássio para avaliação do vigor de sementes de hortaliças: feijão vagem e quiabo. Revista Brasileira de Sementes, v.20, n.2, p.408-413, 1998.

HAMPTON, J.G.; TeKRONY, D.M. (Eds.). INTERNATIONAL SEED TESTING ASSOCIATION ISTA. Handbook of vigour test methods. 3rd ed. $117 \mathrm{p}$., 1995.

LOOMIS, E.L., SMITH, O.E. The effect of artificial ageing on the concentration of $\mathrm{Ca}, \mathrm{Mg}, \mathrm{Mn}, \mathrm{K}$, and $\mathrm{Cl}$ in imbibing cabbage seed. Journal of Americam society of Horticultural Science, v.105, n.5, p.647-650, 1980.

MAGUIRE, J.D. Speed of germination aid in selection and evaluation for seedling na vigour. Crop Science, Madison, v.2, n.2, p.176-177, 1962.

MARCOS FILHO, J. Fisiologia de sementes de plantas cultivadas. Piracicaba: Fealq, 2005. 495p.

MARCOS FILHO, J. Teste de envelhecimento acelerado. In: KRZYZANOWSKI, F.C.; VIEIRA, R.D.; FRANÇA NETO, J.B. (Ed.). Vigor de sementes: conceitos e testes. Londrina: ABRATES, 1999b. cap.3, p.1-24.

MARCOS FILHO, J. Testes de vigor: importância e utilização. In: KRZYZANOWSKI, F.C.; VIEIRA, R.D.; FRANÇA NETO, J.B. (Ed.). Vigor de sementes: conceitos e testes. Londrina: ABRATES, 1999a. cap.1, p.1-21.

MIGUEL, M.V.C.; MARCOS FILHO, J. Potassium leakage and maize seed physiological potential. Scientia Agrícola, Piracicaba, v.59, n.2, p.315-319, 2002.

MIRANDA, D.M.; NOVEMBRE, A.D.L.C.; CHAMMA, H.M.C.P.; MARCOS FILHO, J. Avaliação do potencial fisiológico de sementes de pimentão pelo teste de lixiviação de potássio. Informativo Abrates, v.13, n.3, p.275, 2003.

PANOBIANCO, M.; MARCOS FILHO, J. Evaluation of the physiological potential of tomato seeds by germination and vigor tests. Seed Technology, Lansing, v.23, n.2, p.151-161, 2001.

POWELL, A.A. Cell membranes and seed leachate conductivity in relation to the quality of seed for sowing. Journal of Seed Technology, Fort Collins, v.10, n.2, p.81$100,1986$.

RODO, A.B.; MARCOS FILHO, J. Teste de lixiviação de potássio para avaliação rápida do potencial fisiológico de sementes de cebola. Informativo Abrates, Londrina, v.11, n.2, p.183, 2001. 
SIMON, E.W.; RAJA-HARUN, R.M. Leakage during seed imbibition. Journal of Experimental Botany, v.23, n.77, p.1076-1085, 1972.

TAYLOR, A.G.; LEE, S.S; BERESNIEWICZ, M.M.; PAINE, D.H. Amino acid leakage from aged vegetable seeds. Seed Science and Technology, v.23, n.1, p.113$122,1995$.
VANZOLINI, S.; NAKAGAWA, J. Lixiviação de potássio na avaliação da qualidade fisiológica de sementes de amendoim. Revista Brasileira de Sementes, v.25, n.2, p.7-12, 2003.

WOODSTOCK, L.W.; FURMAN, K.; LEFFLER, H.R. Relationship between weathering deterioration and germination, respiratory metabolism, and mineral leaching from cottonseeds. Crop Science, v.25, n.3, p.459-466, 1985. 